\title{
Pharyngeal-cervical-brachial variant of Guillain-Barré syndrome
}

INSERM

\section{Source}

INSERM. (1999). Orphanet: an online rare disease and orphan drug data base.

Pharyngeal-cervical-brachial variant of Guillain-Barré syndrome. ORPHA:231426

Pharyngeal-cervical-brachial variant of Guillain-Barré syndrome is a rare, acquired peripheral neuropathy disease characterized by rapidly progressive oropharyngeal (facial palsy, dysarthria) and cervicobrachial weakness, associated with upper limb weakness and hypo/areflexia, in the absence of ophthalmoplegia, ataxia, altered consciousness, and prominent lower limb weakness. The presence of monospecific IgG anti-GT 1a antibodies is associated. 